\title{
Piloplex, a new long-acting pilocarpine polymer salt. A: Long-term study
}

\author{
URIEL TICHO, ${ }^{1}$ MICHAEL BLUMENTHAL, ${ }^{2}$ SHLOMO ZONIS, ${ }^{3}$ ALON GAL, ${ }^{4}$ \\ IZHAK BLANK, ${ }^{4}$ AND ZEEV W. MAZOR \\ From the ${ }^{1}$ Department of Ophthalmology, Hadassah Hospital, Jerusalem, the ${ }^{2}$ Department of Ophthalmology, \\ Sheba Hospital, Tel-Hashomer, the ${ }^{3}$ Department of Ophthalmology, Rambam Hospital, Haifa, and \\ ${ }^{4}$ Hydrophilics Laboratories, Haifa, Israel
}

SUMMARY Thirty eyes of 15 patients with open-angle glaucoma were followed up for a period of up to 1 year while being treated with Piloplex eye drops containing a new long-acting pilocarpine polymer salt. Average morning intraocular pressure (IOP) values during treatment with pilocarpine hydrochloride administered 4 times daily was $20.5 \mathrm{mmHg}$. Average morning IOP values during Piloplex medication administered only twice daily were 19.8 to $18.2 \mathrm{mmHg}$ (range of averages on 14 sessions). These findings indicate the lower average pressure during Piloplex medication and show its prolonged hypotensive effect. Both medications contained an equivalent total daily amount of pilocarpine. Throughout the 1-year study period no adverse side effects were reported, and only 1 patient complained of local sensitivity reaction. Visual disturbances characteristic of pilocarpine eye drops were reduced from 3 times a day on pilocarpine hydrochloride 4 times daily to once a day on Piloplex twice daily.

One of the major problems in the treatment of glaucoma is the limited therapeutic effect (6 to 8 hours) of pilocarpine eye drops. Consequently many attempts have been made to prolong the hypotensive effect of pilocarpine. Increase in concentration of pilocarpine above $4 \%$ has failed to show a significant increase or prolongation of therapeutic effect (Harris and Galin, 1970; Drance and Nash, 1971). Also, increased viscosity of the ophthalmic solution administered only twice daily did not give adequate control for clinical usefulness (Quigley and Pollack, 1977).

A prolongation of therapeutic effect, however, can be achieved by sustained release of pilocarpine to the ocular tissues. This is possible only when release of the drug from its vehicle is rate-limiting (Sieg and Robinson, 1977).

Piloplex emulsion, which contains a new pilocarpine polymer salt, has been designed to give sustained release of pilocarpine. This characteristic of Piloplex has been demonstrated in vitro by comparing the release patterns of pilocarpine hydrochloride with Piloplex. The results, summarised in Fig. 1, indicated that the release time of $80 \%$ of the

Address for reprints: Dr U. Ticho, Department of Ophthalmology, Hadassah Hospital, Jerusalem, Israel

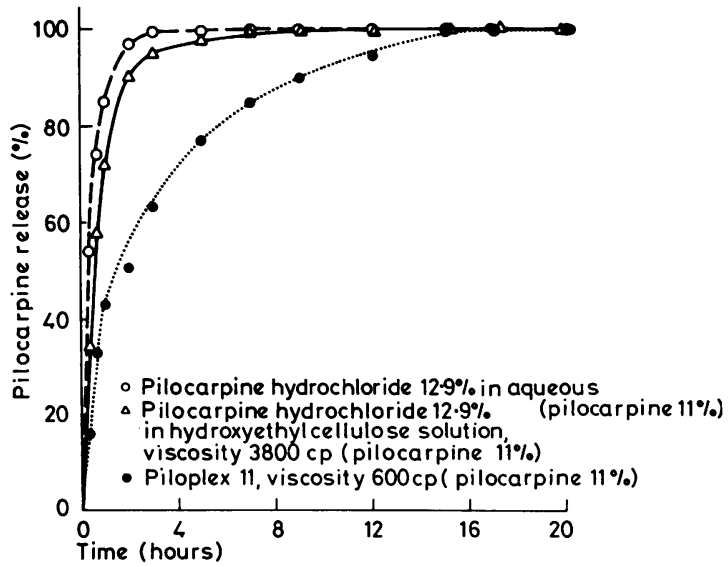

Fig. 1 In vitro release of pilocarpine from Piloplex and pilocarpine hydrochloride solutions from a dialysis bag. The medium was an agitated isotonic saline solution (pH 7.1) maintained at $37^{\circ} \mathrm{C}$. Active ingredient was determined spectrophotometrically (Ticho et al., 1978)

active ingredient from Piloplex is 6 hours, against 1 hour for the corresponding pilocarpine hydrochloride solutions.

Preliminary clinical trials on 68 patients with glaucoma treated with Piloplex confirmed its 
prolonged hypotensive effect (Ticho et al., 1978; Blumenthal et al., 1979). In these studies Piloplex administered twice daily induced a lower average diurnal intraocular pressure (IOP) level with fewer IOP fluctuations than pilocarpine hydrochloride administered 4 times daily (Fig. 2). The total daily amount of pilocarpine applied in the two different medications was equal. The present paper reports on the therapeutic effect and safety of long-term Piloplex medication.

\section{Patients and methods}

The study was carried out at 3 different glaucoma units. At the Hadassah, Sheba, and Rambam hospitals in Israel 30 eyes of 15 patients who agreed to participate in this survey with their informed consent were studied. All patients had bilateral open-angle glaucoma with visual field defects. All had been under our medical supervision for at least 2 years before this study. Ten of our patients were males and 5 were females. They were aged 48 to 84 except for 1 patient who was 18 years old. The study was divided into two periods: (1) Two-week period in which the patients were treated with their prestudy medications. These medications consisted of pilocarpine hydrochloride (PHCl) $2 \%$ or $4 \% 4$ times daily, which contains 1.7 and $3.4 \%$ pilocarpine base. To 5 patients additional epinephrine borate $(1 \%$ of free base) and/or acetazolamide $250 \mathrm{mg}$ were administered twice a day. The detailed prestudy

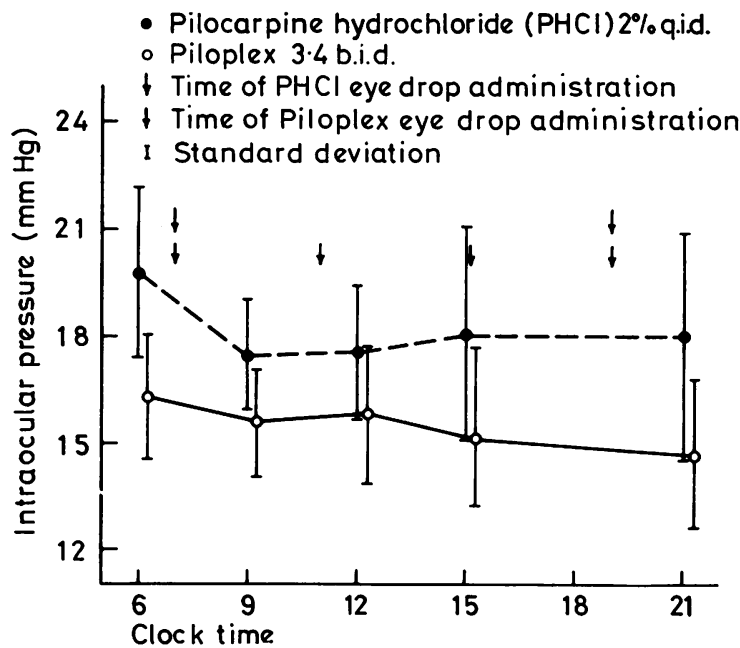

Fig. 2 Average diurnal IOP curve of 21 eyes $(12$ hospitalised patients) on the third day of pilocarpine hydrochloride $2 \% 4$ times daily treatment and on the third day of Piloplex 3.4 twice daily treatment (Blumenthal et al., 1979)
Table 1 Patients and medication data

\begin{tabular}{|c|c|c|c|c|c|}
\hline \multicolumn{3}{|c|}{ Patients } & \multirow{2}{*}{$\begin{array}{l}\text { Prestudy } \\
\text { medicine } \\
\text { (period A) }\end{array}$} & \multirow{2}{*}{$\begin{array}{l}\text { Study } \\
\text { medicine } \\
(\text { period } \mathrm{B})\end{array}$} & \multirow{2}{*}{$\begin{array}{l}\text { Study } \\
\text { duration } \\
\text { (months) }\end{array}$} \\
\hline No. & Age & Sex & & & \\
\hline 1 & 65 & $\mathbf{F}$ & PHCl 2 & PX 3.4 & 12 \\
\hline 2 & 66 & $\mathbf{M}$ & PHCl 2 & PX 3.4 & 8 \\
\hline 3 & 78 & $\mathbf{M}$ & PHCl 2 & PX 3.4 & 12 \\
\hline 4 & 76 & $\mathbf{M}$ & PHCl 2 & PX 3.4 & 8 \\
\hline 5 & 65 & $\mathbf{M}$ & PHCl 2 & PX 3.4 & 11 \\
\hline 6 & 73 & $\mathbf{M}$ & PHCl 2, E & PX $3.4, E$ & 9 \\
\hline 7 & 18 & $\mathbf{F}$ & PHCl 2, E, AC & PX $3.4, E, A C$ & 8 \\
\hline 8 & 54 & $\mathbf{M}$ & PHCl 4 & PX 6.8 & 12 \\
\hline 9 & 61 & $\mathbf{M}$ & PHCl 4 & PX 6.8 & 12 \\
\hline 10 & 65 & $\mathbf{F}$ & PHCl 4 & PX 6.8 & 12 \\
\hline 11 & 49 & $\mathbf{F}$ & PHCl 4 & PX 6.8 & 12 \\
\hline 12 & 69 & $\mathbf{M}$ & PHCl 4, AC & PX 6.8, AC & 12 \\
\hline 13 & 52 & $\mathbf{M}$ & PHCl 4, E, AC & PX 6.8, E, AC & 8 \\
\hline 14 & 84 & $\mathbf{F}$ & PHCl 4, E, AC & PX 6.8, E, AC & 9 \\
\hline 15 & 60 & $\mathbf{M}$ & PHCl 4, E, AC & PX 68 , E, AC & 12 \\
\hline
\end{tabular}

Abbreviations for medications administered are as follows: $\mathrm{PHCl}-$ pilocarpine hydrochloride given 4 times daily (number following $\mathrm{PHCl}$ indicates the percentage of $\mathbf{P H C l})$. PX - Piloplex given twice daily (number following PX indicates the percentage of pilocarpine). $\mathrm{E}$ - epinephrine borate ( $1 \%$ of free base) given twice daily. AC acetazolamide $250 \mathrm{mg}$ given twice daily.

medication is listed in Table 1. (2) After the first 2 weeks of treatment $\mathrm{PHCl} 2$ or $4 \%$ was replaced by Piloplex 3.4 and 6.8 twice daily respectively. Piloplex 3.4 and 6.8 contains 3.4 and $6.8 \%$ pilocarpine.

Morning intraocular pressures were measured by the Goldmann applanation tonometer before medications on day 14 of period $A$ and on days 7 , 14 , and 28 and every month thereafter through 8 to 12 months of period $B$. At each visit tolerance and side effects were recorded. Visual fields were obtained every 3 months.

\section{Results}

Out of the 30 eyes studied $26(87 \%)$ had an IOP of less than $24 \mathrm{mmHg}$ throughout all measurements over an 8- to 12-month period. None of the patients presented progression in field loss. Average morning IOP of the 30 eyes at the end of the first 2 weeks of PHCl 4 times daily (period $A$ ) was $20.5 \mathrm{mmHg}$. This value was higher than those measured during the 8 to 12 months of Piloplex twice daily (period $B$ ) (range of average morning IOP values: 19.8 to 18.2 $\mathrm{mmHg}$ ). The average morning IOP curve throughout 


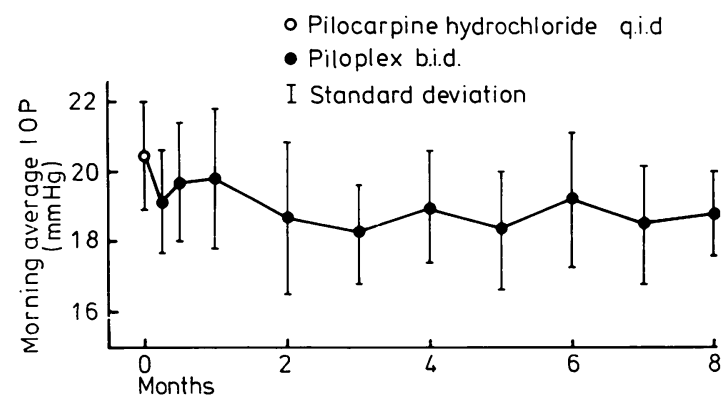

Fig. 3 Average morning IOP curve of the 30 eyes studied during pilocarpine hydrochloride 4 times daily medication (period A) followed by Piloplex twice daily treatment (period B)

the study period is illustrated in Fig. 3. In the 5 patients to whom epinephrine drops were administered in addition to Piloplex an anticipated additive effect was observed, similar to that found with the use of $\mathrm{PHCl}$ and epinephrine.

No signs of ocular damage followed Piloplex medication. Specifically, no corneal abrasions, conjunctival reactions, or inflammations were observed. One case was excluded owing to a local sensitivity reaction (No. 13), and 1 (No. 4) died of an unrelated cause. Those patients who had undesirable myopia, induced by miotic drugs following Piloplex administration (10/15), reported that Piloplex caused a similar effect to that caused by PHCl during period $A$. All patients reported that on the Piloplex regimen blurring occurred only once a day (morning dose) instead of 3 times a day while on pilocarpine hydrochloride 4 times daily.

\section{Discussion}

In our previous study we demonstrated the slow release of pilocarpine ion from the pilocarpine polymer salt compound Piloplex (Ticho et al., 1978). In further clinical studies (Ticho et al., 1978; Blumenthal et al., 1979) Piloplex emulsion was found to be more effective in maintaining round-the-clock IOP than PHCl. Moreover, Piloplex was better than $\mathrm{PHCl}$ in maintaining a significantly lower mean IOP with less variation in diurnal values. Furthermore, these results were achieved by 2 applications per day of Piloplex instead of 4 applications of $\mathrm{PHCl}$.

In this long-term study Piloplex was shown to be a safe drug which maintains its hypotensive effects. Systemic side effects were not present, and local sensitivity was found only in 1 patient out of the 15 studied. Over the long-term period the patients became accustomed to this medication, and subjectively preferred it to $\mathrm{PHCl}$, mainly because of the twice daily regimen. Adverse visual disturbances were also reported to occur less owing to the fewer daily applications of Piloplex. In conclusion, this study has shown the long-term therapeutic effect and safety of Piloplex medication throughout a trial period of 1 year.

\section{References}

Blumenthal, M., Ticho, U., Zonis, S., Gal, A., Blank, I., and Mazor, Z. W. (1979). Further clinical trial with Piloplex-a new long acting pilocarpine salt. Annals of Ophthalmology (in Press).

Drance, S. M., and Nash, P. A. (1971). The dose response of human intraocular pressure to pilocarpine. Canadian Journal of Ophthalmology, 6, 9-13.

Harris, L. S., and Galin, M. A. (1970). Dose response analysis of pilocarpine induced ocular hypotension. Archives of Ophthalmology, 84, 605-608.

Quigley, A. H., and Pollack, I. P. (1977). Intraocular pressure control with twice daily pilocarpine in two vehicle solutions. Annals of Ophthalmology, 9, 427-429.

Sieg, J. W., and Robinson, J. R. (1977). Vehicle effects on ocular drug bioavailability. II. Evaluation of pilocarpine. Journal of Pharmaceutical Science, 66, 1222-1228.

Ticho, U., Blumenthal, M., Zonis, S., Gal, A., Blank, I., and Mazor, Z. W. (1978). A new long acting pilocarpine compound. Annals of Ophthalmology (in press). 